\title{
Vascularização múltipla da artéria renal: estudo anatômico de cadáveres humanos
}

\section{Supranumeric vascularization of renal artery: anatomical study of human corpses}

Ramon Magro Ferreira ${ }^{{ }^{*}}$, Diogo Barros Gutterres ${ }^{\dagger}$, Iago Caetano Cariello ${ }^{\dagger}$, Paulo Víctor Innocencio Póvoa de Castro ${ }^{\dagger}$, Valdir Donizeti Alves Junior ${ }^{\dagger}$, Emílio Conceição de Siqueira

Como citar esse artigo. Ferreira, R.M.; Gutterres, D.B.; Cariello, I.C.; de Castro, P.V.I.P.; Junior, V.D.A.; de Siqueira, E.C. Vascularização múltipla da artéria renal: estudo anatômico de cadáveres humanos. Revista de Saúde. 2021 Dez./Mar.; 12 (1): XX-XX

\section{Resumo}

A irrigação é de vital importância para a sobrevivência de qualquer tecido. Cada rim é suprido por uma única artéria, denominada renal principal. A presença de múltiplas artérias renais, assim como o padrão de suas divisões pré-hilares, deve ser avaliada devido à importância em relação à irrigação renal. Sendo assim, as variações da artéria renal estão se tornando mais importantes devido ao aumento gradual dos procedimentos radiológicos intervencionistas, das operações urológicas, vasculares e do transplante renal. O objetivo do estudo é conhecer a prevalência de variações múltiplas das artérias renais no anatômico da Universidade de Vassouras além de revisar a literatura, atualizando os profissionais de saúde e estudantes. Foram utilizados para a execução do trabalho 51 rins, sendo 29 esquerdos e 22 direitos. Observouse múltiplas artérias em $25,5 \%$ rins que faziam parte da amostra do estudo, no qual $76,9 \%$ dessas foram do lado direito e 23,1\% esquerdo. Grande parte dos resultados encontrados nesse estudo está de acordo com a literatura, entretanto, nessa pesquisa observou-se que houve prevalência de variações encontradas no rim direito, divergindo das literaturas. O conhecimento das variações encontradas nos vasos renais é de suma importância para a sistematização da anatomia radiológica, cirurgia geral e ensino. Conclui-se que variações renais podem expressar uma influência significativa na predisposição à enfermidade, curso da doença, achados nos exames clínicos ou até mesmo na conduta do paciente, além de influenciar procedimentos cirúrgicos.

.Palavras-chave: variação anatômica; artéria renal.

\begin{abstract}
Irrigation is of vital importance for the survival of any tissue. Each kidney is supplied by a single artery, called the main renal. The presence of multiple renal arteries, as well as the pattern of their prehilar divisions, should be evaluated because of the importance of renal irrigation. Thus, variations in the renal artery are becoming more important due to the gradual increase in interventional radiological procedures, urological, vascular and renal transplantation operations. The objective of the study is to know the prevalence of multiple variations of the renal arteries in the anatomical of the University of Vassouras in addition to reviewing the literature, updating health professionals and students. 51 kidneys were used for the execution of the work, being 29 left and 22 rights. Multiple arteries were observed in $25.5 \%$ kidneys that were part of the study sample, where $76.9 \%$ of these were on the right side and $23.1 \%$ left. Most of the results found in this study are in agreement with the literature, however, in this research it was observed that there was prevalence of variations found in the right kidney, diverging from the literature. The knowledge of the variations found in the renal vessels is of paramount importance for the systematization of the radiological anatomy, general surgery and teaching. It is concluded that renal variations can express a significant influence on the predisposition to the disease, course of the disease, findings in clinical exams or even on the patient's behavior, besides influencing surgical procedures.

Keywords: anatomic variation; renal artery.
\end{abstract}

\section{Introdução}

A irrigação é de vital importância para a sobrevivência de qualquer tecido. ${ }^{1}$ De acordo com as descrições anatômicas, cada rim é suprido por uma única artéria, denominada renal principal. ${ }^{2}$ As artérias renais transportam mais de $20 \%$ do sangue arterial da aorta abdominal. ${ }^{3}$ Usualmente, esses vasos originam-se da aorta ou das artérias ilíacas comuns entre os níveis de T12 e L3. Raramente, eles podem originar-se da artéria suprarrenal, do tronco celíaco, da mesentérica superior, da mesentérica inferior, da sacral mediana e da frênica inferior. ${ }^{2,4}$ A artéria renal direita é mais cranial que a esquerda, em conformidade com a posição relativamente mais cranial do rim. ${ }^{2,3}$

O desenvolvimento do sistema urogenital é altamente complicado em seres humanos. Portanto, as anomalias congênitas ou as variações são maiores em comparação com as de outros sistemas no mesmo indivíduo. ${ }^{5}$ As variações da artéria renal são divididas 
em 2 grupos: divisão precoce e artéria renal acessória (ARA). A ramificação das principais artérias renais em ramos segmentares mais proximal do que a nível do hilo renal, é chamada de divisão precoce. ARA é dividida em dois grupos: as artérias hilares (acessórias) entram nos rins do hilo com a artéria renal principal, enquanto as artérias polares (aberrantes) entram nos rins diretamente da cápsula fora do hilo. ${ }^{5,6} \mathrm{~A}$ presença de múltiplas artérias renais, assim como o padrão de suas divisões pré-hilares devem ser avaliados, devido à importância em relação à irrigação renal e por influenciar nos planos de dissecção e acesso ao hilo renal. ${ }^{7}$

As descrições anatômicas da vascularização renal foram apresentadas com resultados diferentes por diversos autores como: Gray descreve a incidência de artéria renal acessória em 23\%, Moore relata o percentual de $25 \%$, Hamilton de até $30 \%$, Riella de 20 a $30 \%$ e Hollinshead refere possibilidade de até $33 \%{ }^{1}$

$\mathrm{O}$ conhecimento das variações da anatomia vascular renal possui grande importância na exploração semiológica renal e outras condições, assim como tratamento do trauma renal, hipertensão renovascular, transplante renal, angioplastia ou reconstrução vascular para lesões congênitas ou adquiridas, cirurgia para aneurisma de aorta abdominal e cirurgia renal. ${ }^{3} \mathrm{O}$ assunto em questão foi escolhido devido a sua relevância que mesmo com a medicina avançada com toda tecnologia disponível, encontra-se uma diversidade de pacientes que não se beneficiam desta e podem estar sujeitos aos riscos e complicações.

O objetivo do estudo foi relatar o nível de prevalência de variações das artérias renais múltiplas do Instituto de Anatomia da Universidade de Vassouras (UV) para melhor conhecimento da vascularização renal e comparar com os achados das atuais literaturas, dados esses relevantes para o emprego cirúrgico rotineiro, exames de imagem, investigação diagnóstica, estudos acadêmicos e transplantes renais.

\section{Material e método}

O estudo foi realizado através da revisão bibliográfica nas bases de dados Pubmed, Lilacs e Scielo, além dos livros Anatomia Orientada para Clínica Moore 2014 e Gray's Anatomia Clínica para Estudantes 2015, com posterior análise comparativa os resultados apresentados com a literatura. Com a supervisão do professor e orientados na literatura do Moore 2014, foram avaliados todos os rins já dissecados de cadáveres formalizados, de ambos os gêneros, diferentes idades e de grupos étnicos distintos, provenientes do Instituto de Anatomia da UV, que se localiza no Campus da Universidade. Utilizaram-se apenas rins com estruturas anatômicas íntegras, incluindo vascularização preservada, sendo assim foram selecionados 51 rins, sendo 29 direitos e 22 esquerdos. Na realização da coleta de dados, cada rim recebeu um adesivo com a numeração de 1 a 51 para que fosse possível a identificação de cada um deles, evitando a existência de dados não fidedignos. Primeiramente foram numerados os rins direitos e, após, os esquerdos. Os rins que apresentavam alguma variação anatômica foram analisados e contabilizados através de gráficos e tabulado no Excel 2010.

\section{Resultados}

Foram utilizados para a execução do trabalho 51 rins previamente dissecados no instituto de Anatomia Humana da UV, sendo 29 rins direito e 22 rins esquerdo. Dentre os rins escolhidos para o estudo, foram encontradas características como artérias renais múltiplas, que envolviam artérias com divisão precoce e ARA - que se subdivide em artérias hilares e artérias polares. Foram observadas múltiplas artérias em 25,5\% $(n=13)$ dos rins que faziam parte da amostra do estudo (Figura 1). Em relação às características das variantes que continham as artérias múltiplas, visualizou-se que $76,9 \%(n=10)$ dessas foram do lado direito e $23,1 \%(n=$ 3 ) esquerdo (Figura 2).

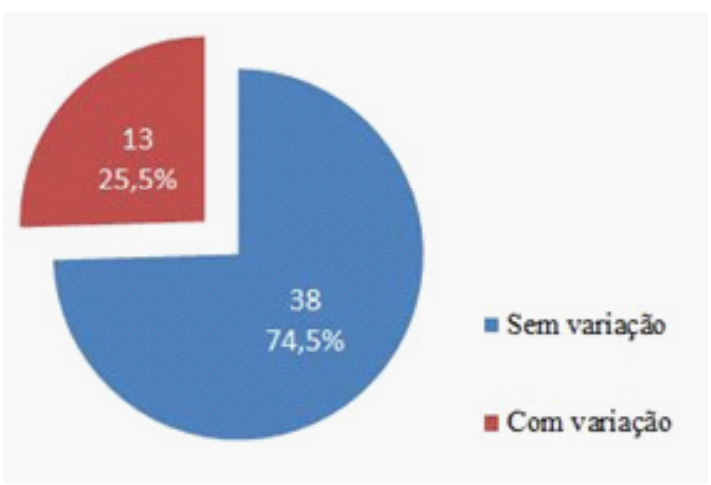

Figura 1. Divisão entre rins com e sem variações anatômicas de múltiplas artérias renais do Instituto de Anatomia Humana da Universidade de Vassouras.

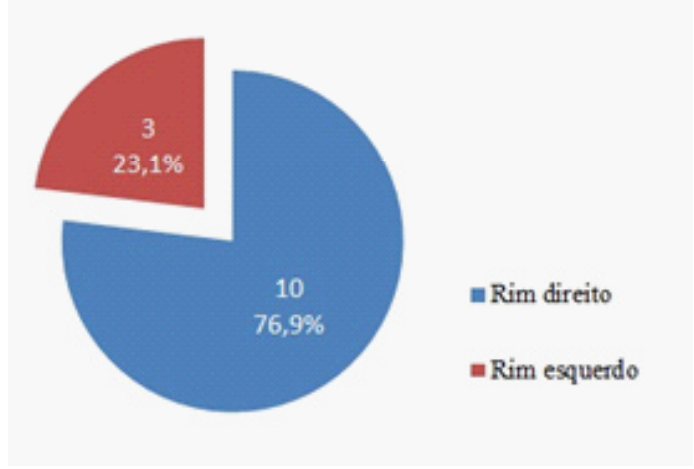

Figura 2. Divisão entre as variações das artérias renais múltiplas nos rins direito e esquerdo do Instituto de Anatomia da Universidade de Vassouras. 


\section{Discussão}

Variação anatômica é definida como uma flexibilidade normal na topografia e morfologia das estruturas do corpo. ${ }^{3}$ As variações das artérias renais estão se tornando mais importantes devido ao aumento gradual dos procedimentos radiológicos intervencionistas, das operações urológicas e vasculares e do transplante renal. ${ }^{6}$ As múltiplas artérias renais aparecem devido à persistência das artérias que surgem da aorta, das artérias ilíacas comuns, das artérias ilíacas internas e dos ramos esplâncicos laterais. Essas artérias persistem devido a não ascensão ou a subida incompleta dos rins à posição normal. ${ }^{5} \mathrm{O}$ domínio da anatomia vascular possibilita ao cirurgião taxas de complicações menores e pode ser um fator fundamental na escolha da melhor técnica operatória, além de possuir grande importância na exploração dos rins para casos de tratamento do traumarenal, transplante renal, hipertensão renovascular, embolização da artéria renal, angioplastia ou reconstrução vascular para lesões congênitas ou adquiridas, cirurgia para aneurisma da aorta abdominal e cirurgia renal conservadora ou radical., 8

As complicações vasculares pós-operatórias foram menores nos casos de rins com uma única artéria versus múltiplas artérias $(12,7$ vs $16,3 \%, \mathrm{P}<0,05) .{ }^{9}$ Mais recentemente, um outro estudo, observou-se que nos enxertos renais de rins com múltiplas artérias não houve problema no centro hospitalar, apesar de necessitar de um maior tempo operatório, maior tempo de isquemia fria e uma maior taxa de transfusão de sangue, sendo posteriormente comparado os resultados a curto e longo prazo entre os grupos. ${ }^{10}$

Aangiografia renal agora é considerada ferramenta não invasiva, rápida e muito importante no estudo da anatomia vascular e suas variações, como duplicidade da artéria renal. Este método é básico para avaliações pré-cirúrgicas, principalmente em intervenções como nefrectomia parcial, laparoscopia e transplante renal. ${ }^{11}$ Variações normalmente não interferem nas funções do organismo. No entanto, podem expressar uma influência significativa na predisposição a enfermidade, curso da doença, achados nos exames clínicos ou até mesmo na conduta do paciente. ${ }^{12}$

Em relação à predisposição a enfermidades, pacientes com artérias renais múltiplas constituem um grupo que possui alta atividade de renina plasmática e que, portanto, pode propiciar o desenvolvimento de hipertensão arterial. ${ }^{13}$ Um estudo realizado por Marshall, no qual foram analisadas 400 peças de necropsia, observou que $80 \%$ dos pacientes eram hipertensos e apenas $49 \%$ dos normotensos havia artéria renal supranumerária (múltiplas artérias renais). ${ }^{14} \mathrm{Na}$ importância cirúrgica foi descrita que nos transplantes renais com presença de múltiplas artérias a taxa de estenose arterial tardia é mais elevada,${ }^{15}$ além do risco aumentado de sangramento quando há dificuldade na realização das anastomoses arteriovenosas, em pacientes com múltiplas artérias e veias renais. ${ }^{9}$

Grande parte dos resultados encontrados nesse estudo está de acordo com a literatura quando a mesma refere que múltiplas artérias renais ocorrem em $20 \%$ a $50 \%$ das pessoas, sendo a sua variação mais comum do que para outros órgãos, ${ }^{16,17}$ Gray diz que há presença da artéria renal acessória em $23 \%$ da população geral, ${ }^{18}$ Moore descreve o percentual de $25 \%,{ }^{19}$ e no trabalho executado no departamento de Anatomia da UV o número de variação encontrado foi de $25,5 \%$. Entretanto, nos seres humanos a observação das artérias renais acessórias (múltiplas) foi mais frequente no rim esquerdo ${ }^{20}$ fato este que não foi observado no presente estudo uma vez que houve prevalência de variações encontradas no rim direito (76,9\%), no entanto observase um viés de seleção ao levantar dados numéricos não compatíveis com peças de um único cadáver, visto que foram selecionadas apenas as estruturas com condições adequadas de estudo.

O conhecimento prévio das variações dos vasos renais pode prevenir lesões inadvertidas durante sua manipulação, contribuindo principalmente nas intervenções cirúrgicas rotineiras, bem como para transplantes renais e estudos radiológicos. Outro aspecto importante do conhecimento da anatomia arterial renal está nos casos de ressecções renais parciais e tumorectomias. ${ }^{21}$ Além disso, a bifurcação arterial préhilar tem fundamental importância em pacientes renais doadores, já que é necessário o comprimento mínimo de $2 \mathrm{~cm}$ da artéria renal antes da bifurcação para garantir uma anastomose adequada. ${ }^{11}$ Para evitar iatrogenia é preciso reconhecimento, estudo e habilidade técnica do profissional envolvido nas mais variadas atividades relacionada à saúde.

Outro assunto que traz muitas discussões nos trabalhos publicado é em relação a nomenclatura da variação anatômica que tange aos vasos renais. Como o termo supranumerária refere a um número acima do esperado, sendo o mais indicado. Além disso, o termo acessório pode nos passar a ideia de que a ligadura destes vasos não iria provocar riscos isquêmicos ao parênquima renal, ${ }^{22}$ o que é um equívoco frequente dos estudantes.

\section{Considerações Finais}

As variações da artéria renal estão se tornando mais importantes devido ao aumento gradual dos procedimentos radiológicos intervencionistas, operações urológicas, vasculares, transplantes renais e experimentais, mediante a prevalência significativa como expresso no estudo, visando minimizar erros 
cometidos decorrentes do desconhecimento, tais como iatrogenia cirúrgica ou diagnóstica. A variação vascular renal é de extrema importância para os cirurgiões que se aproximam dos rins da via retroperitoneal para transplantes renais, procedimentos de embolização, angioplastia e intervenções cirúrgicas após traumas renais. $\mathrm{O}$ presente estudo demonstrou uma frequência de variações anatômicas das artérias renais $(25,5 \%)$ equivalente aos estudos publicados em artigos científicos, divergindo apenas no fato de ter demonstrado maior prevalência no rim direito, o que ressalta a importância de maior preparo do médico cirurgião, radiologista ou clínico na hora de abordar esses pacientes para evitar erros diagnósticos e terapêuticos. O conhecimento das variações encontradas nos vasos renais é de suma importância para a sistematização da anatomia radiológica, cirúrgica e ensino. Conclui-se que podem expressar uma influência significativa na predisposição a enfermidade, curso da doença, achados nos exames clínicos ou até mesmo na conduta do paciente, além de influenciar procedimentos cirúrgicos.

\section{Referências Bibliográficas}

1. Sebben GA, Rocha SL, Quidigno E, Caliari E, Caliari H. Variações das artérias renais: estudo anatômico em cadáveres. Rev Med Res. 2011; 13 (4): $245-50$.

2. Palmieri BJ, Petroianu A, TCBC-MG, Silva L, Andrade L, Alberti LR. Estudo do padrão arterial de 200 pedículos renais por meio de angiotomografias. Rev Col Bras Cir.(Rio de Janeiro). 2011; 38 (2): 116-21

3. Figueiredo M, Roza M, Passos N, Silva B, Scherer P. Artéria renal com dupla origem na porção abdominal da aorta em caprino. Acta Veterinaria Brasilica. 2009; 3(1): 38-42

4. Mir N, Hassan A, Rangrez R, Hamid S, Sabia, Tabish SA, et al. Bilateral Duplication of Renal Vessels: Anatomical, Medical and Surgical perspective. Int J Health Sci. 2008; 2(2): 179-185

5. Krishnaveni C, Kulkarni R. A Right Ectopic Kidney with Bilateral Multiple Anomalies of the Renal Vasculature - A Case Report. J Clin Diagn Res. 2013; 7(1): 150-3.

6. Ozkan U, Oğuzkurt L, Tercan F, Kizilkiliç O, Koç Z, Koca N. Renal artery origins and variations: angiographic evaluation of 855 consecutive patients. Diagn Interv Radiol. 2006; 12(4):183-6.

7. Stocco AV, Ferreira LT, Sousa CAS, Figueiredo MA. Artéria renal esquerda múltipla em gato sem raça definida: relato de caso. Acta Vet Bras. 2016; 10(1): 74-8

8. Júnior W, Filho J. Estudo da Distribuição Arterial em Rins Humanos. Arquivos Catarinenses de Medicina. 2003; 32(26): 21-7

9. Araújo JC, Neves CL. Impacto clínico das complicações cirúrgicas em pacientes transplantados renais em hospital de referência em Salvador. 2015. Trabalho de Conclusão de Curso - Faculdade de Medicina da Bahia, UFBA, Bahia, 2016.

10. Carvalho J, et al. Artérias renais múltiplas na transplantação renal: será um problema atualmente. Congresso da Associação Portuguesa de Urologia, Alfândega do Porto. 22-24 Setembro, 2017, Porto.

11. Sabec-pereira DK, Campos TM, Gutierrez OB, Lima FC, Santa Rita RM, Pereira KF. Double renal artery in human cadavers : a case report. Biosci J. 2017; 33(6): 1666-70

12. Willam PL, Humpherson JR. Concepts of variation and normality in morphology: important issues at risk of neglect in modern undergraduate medical courses. Clin. Anat. 1999; 12:186-190.

13. Glodny B, Cromme S, Reimer P, et al. Hypertension associated with multiple renal arteries may be renin-dependent. J Hypertens. 2000; 18(10): 1437-44.

14. Marshall AG. Aberrant renal arteries and hypertension.Lancet 1951; 2:701-5.

15. Benedetti E, Troppman C, Gillingham K, Sutherland DE, Payne WD, Dunn DL, Matas AJ, Najarian JS, Grussner RW. Short- and long-term outcomes of kidney transplants with multiple renal arteries.Ann Surg 1995; 221(4):406-14

16. Harrison LH Jr, Flye MW, Sleigler HF. Incidence of anatomical variants in renal vasculature in the presence of normal renal function. Ann Surg 1978; 188(1):83-9.

17. Sampaio FJ, Passos MA. Renal arteries: anatomic study for surgical and radiological practice. SurgRadiolAnat 1992; 14(2):113-7.

18. Gray H, Goss CM. Anatomia. $29^{\mathrm{a}}$ ed. Rio de Janeiro: Guanabara Koogan; 1988.

19. Moore, KL. Anatomia orientada para a clínica. 7. ed. Rio de Janeiro: Guanabara Koogan, 2014

20. Karmacharya J, Parmer SS, Antezana JN, Fairman RM, Woo EY, Velazquez OC, Golden MA, Carpenter JP.2006. Outcomes of accessory renal artery occlusion during endovascular aneurysmrepair. J. Vasc. Surg. 43:8-13.

21. Sampaio FJB. Sistematização pielocalicial: morfometria renal e moldagem das cavidades coletoras com resina de poliéster. [Dissertação de Mestrado] Rio de Janeiro: UFRJ; Departamento de Anatomia. 1986.

22. Sampaio FJB. Renal arterial pedicle - anatomic analysis applied to urologic and radiologic procedures. In: Sampaio FJB, Uflacker R. Renal anatomy applied to urology, endourology, and interventional radiology. Thieme Medical Publishers. New York: p.47-54, 1993 